\title{
New pseudoclassical model for Weyl particles
}

\author{
D. M. Gitman, A. E. Gonçalves and I. V. Tyutin * \\ Instituto de Física, Universidade de São Paulo P.O. Box 20516, 01452-990 São Paulo, \\ São Paulo, Brazil
}

(June 28, 2021)

\begin{abstract}
A new pseudoclassical model to describe Weyl particles is proposed. Different ways of its quantization are presented. They all lead to the theory of Weyl particle; namely, the massless Dirac equation and the Weyl condition are reproduced. In contrast with models discussed previously, this one admits both the Dirac quantization and quasicanonical quantization, with the fixation of almost all gauge freedom on the classical level.

PACS numbers(s):11.10Ef, 03.65.Pm
\end{abstract}

Typeset using REVTEX

*Permanent Address: P. N. Lebedev Institute, 53 Leninsky Pr., Moscow 117924, Russia 
In recent years numerous classical models of relativistic particles and superparticles have been discussed intensively in different contexts. First, the interest in such models was initiated by the close relationship with problems in string theory and gravity, but now it is clear that it is an important problem itself whether there exist classical model for any relativistic particle whose quantization reproduces, in a sense, the corresponding field theory, or one particle sector in the corresponding quantum field theory. In this paper we propose a new pseudoclassical model whose quantization reproduces the quantum theory of Weyl particles. The history of the question is the following. As is known, first, a pseudoclassical action of spin $-\frac{1}{2}$ relativistic particle was proposed by Berezin and Marinov [1] and after that was discussed and investigated in many papers [2- 6]. The action has the form

$$
S=\int_{0}^{1}\left[-\frac{1}{2 e}\left(\dot{x}^{\mu}-i \psi^{\mu} \chi\right)^{2}-\frac{e}{2} m^{2}-i m \psi^{5} \chi-i\left(\psi_{\mu} \dot{\psi}^{\mu}-\psi_{5} \dot{\psi}_{5}\right)\right] d \tau
$$

where $x^{\mu}, e$ are even and $\psi^{\mu}, \chi$ are odd variables, dependent on a parameter $\tau \in[0,1], \mu=$ $\overline{0,3}, \eta_{\mu \nu}=\operatorname{diag}(1-1-1-1)$. Because of the reparametrization invariance of the action, the Hamiltonian of the model is equal to zero on the constraint surface. In Refs. [7 [10, devoted to the quantization of the model, the authors tried to avoid this difficulty, using the so called Dirac method of quantization of theories with first-class constraints [11]. In this method one treats the first-class constraints in the sense of restrictions on state vectors. Unfortunately, in the general case, this scheme of quantization creates many questions, e.g. with Hilbert space construction, the nature of the Schrödinger equation and so on. A consistent, but more complicated technical way is to work in the physical sector, namely, first, on the classical level, to impose gauge conditions to all first class-constraints to reduce the theory to one with second-class constraints only, and then quantize by means of the Dirac brackets (we will call such a method canonical quantization). First canonical quantization for a relativistic spin $-\frac{1}{2}$ particle was done in 12 . The quantum mechanics constructed there admits the limit $m=0$. As a result one gets the quantum theory of massless particles, which is described by the Dirac equation with $m=0$, but without any additional restrictions on the four-spinor $\Psi(x)$ ( see for example 13, 14): 


$$
i \partial_{\mu} \gamma^{\mu} \Psi(x)=0, \quad\left[\gamma^{\mu}, \gamma^{\nu}\right]_{+}=2 \eta^{\mu \nu}
$$

It turns out that the variable $\psi^{5}$ can be omitted from the action (11) at $m=0$. The quantization of such a modified action reproduces the physical sector in the limit $m=0$ of the massive quantum mechanics. Unfortunately, such a quantum theory describes massless spin- $\frac{1}{2}$ particle with all the possible values of helicity (right and left neutrinos). As is known, the right (left) neutrino is described by a four-spinor, which obeys, in addition to the Dirac equation (2), the Weyl condition as well:

$$
\left(\gamma^{5}-\alpha\right) \Psi(x)=0, \quad \alpha=1(-1), \quad \gamma^{5}=i \gamma^{0} \gamma^{1} \gamma^{2} \gamma^{3} .
$$

There were several attempts to modify the action (1) at $m=0$ so that in course of quantization one can get quantum mechanics with wave functions obeying both Eqs. (2) and (3) at the same time. So, in Ref. [10 the authors proposed the action (we are using our notations for this action)

$$
S=\int_{0}^{1}\left[-\frac{1}{2 e}\left(\dot{x}^{\mu}+g_{1} Q^{\mu}-i \psi^{\mu} \chi\right)^{2}-i \psi_{\mu} \dot{\psi}^{\mu}+g_{2}\left(\Lambda-\frac{\alpha}{2}\right)\right] d \tau,
$$

where $g_{a}, a=1,2$ are Lagrange multipliers, $\Lambda=i \epsilon^{\mu \nu \rho \varsigma} \psi_{\mu} \psi_{\nu} \psi_{\rho} \psi_{\varsigma} / 3, Q^{\mu}=\epsilon^{\mu \nu \rho \varsigma} \psi_{\nu} \psi_{\rho} \psi_{\varsigma}$. Quantization by means of the Dirac method gives both Eqs. (22) and (3) as restrictions on state vectors. Namely, the theory has, in particular, second-class constraints $P_{\mu}+i \psi_{\mu}=0$, where $P_{\mu}$ are momenta conjugated to $\psi^{\mu}$, and first-class constraints $\pi^{2}=0$, $\pi_{\mu} \psi^{\mu}=0, \pi_{\mu} Q^{\mu}=0, \Lambda-\alpha / 2=0$, where $\pi_{\mu}$ are momenta conjugated to $x^{\mu}$. Calculating the Dirac brackets with respect to the second class constraints only, one can find in the course of quantization the following realization for essential variables $\hat{\pi}_{\mu}=-i \partial_{\mu}, \quad \hat{\psi}^{\mu}=\frac{i}{2} \gamma^{\mu}$, in the $x$ representation. Applying the first-class constraints operators to the state vector, according to Dirac, one gets only two independent equations

$$
\hat{\pi}_{\mu} \hat{\psi}^{\mu} \Psi(x)=0, \quad\left(\hat{\Lambda}-\frac{\alpha}{2}\right) \Psi(x)=0,
$$

which are just Eqs. (2) and (3). As mentioned before, this way of quantization is not well grounded. Moreover, attempts to quantize this action canonically fail, since as soon 
as one chooses any gauge condition linear in $\psi$, the combination $\Lambda$ vanishes, and only the Dirac equation remains after quantization. Another possibility was discussed in [15]. They considered the theory with the action

$$
S=\int_{0}^{1}\left\{-\frac{1}{2 e}\left[\dot{x}^{\mu}-i\left(\psi^{\mu}-\frac{2 i \alpha}{3} \epsilon^{\mu \nu \rho \varsigma} \psi_{\nu} \psi_{\rho} \psi_{\varsigma}\right) \chi\right]^{2}-i \psi_{\mu} \dot{\psi}^{\mu}\right\} d \tau .
$$

A formal quantization of the theory following the Dirac method leads to the equation $i \partial_{\mu} \gamma^{\mu}\left(\gamma^{5}-\alpha\right) \Psi(x)=0$ for state vectors, which is not equivalent to both Eqs. (2), (3)). The canonical quantization gives the Dirac equation (2), but without any additional restrictions for helicity. That is in agreement with the fact that classically actions (5) and (1) are equivalent at $m=0$ [15].

In this paper we propose a new pseudoclassical action to describe the Weyl particle which is a covariant generalization of an action [16]. It admits both quasicanonical quantization (we are fixing gauge freedom which corresponds to two types of gauge transformations of existing three ones, see below) and the Dirac quantization. Both of them lead to the theory of Weyl particles. The new action has the form

$$
S=\int_{0}^{1}\left[-\frac{1}{2 e}\left(\dot{x}^{\mu}-i \psi^{\mu} \chi-\epsilon^{\mu \nu \rho \varsigma} b_{\nu} \psi_{\rho} \psi_{\varsigma}+\frac{i \alpha}{2} b^{\mu}\right)^{2}-i \psi_{\mu} \dot{\psi}^{\mu}\right] d \tau
$$

where $x^{\mu}, e, \psi^{\mu}$, and $\chi$ have the same meaning as in (11), the variables $b^{\mu}$ form an even four-vector, and $\alpha$ is an even constant.

There are three types of gauge transformations under which the action (6) is invariant: reparametrization

$$
\delta x^{\mu}=\dot{x}^{\mu} \xi, \quad \delta e=\frac{d}{d \tau}(e \xi), \delta b^{\mu}=\frac{d}{d \tau}\left(b^{\mu} \xi\right), \delta \psi^{\mu}=\dot{\psi}^{\mu} \xi, \quad \delta \chi=\frac{d}{d \tau}(\chi \xi)
$$

with an even parameter $\xi(\tau)$; supertransformation

$$
\begin{aligned}
& \delta x^{\mu}=i \psi^{\mu} \epsilon, \quad \delta e=i \chi \epsilon, \quad \delta b^{\mu}=0, \quad \delta \psi^{\mu}=\frac{1}{2 e} z^{\mu} \epsilon, \delta \chi=\dot{\epsilon}, \\
& z^{\mu}=\dot{x}^{\mu}-i \psi^{\mu} \chi-\epsilon^{\mu \nu \rho \varsigma} b_{\nu} \psi_{\rho} \psi_{\varsigma}+\frac{i \alpha}{2} b^{\mu},
\end{aligned}
$$

with an odd parameter $\epsilon(\tau)$; and an additional [in comparasion with the action (11)] gauge transformation 


$$
\begin{aligned}
\delta x^{\mu} & =\left(\epsilon^{\mu \nu \rho \varsigma} b_{\nu} \psi_{\rho} \psi_{\varsigma}-\frac{i \alpha}{2} b^{\mu}\right) \kappa, \quad \delta e=0, \quad \delta b^{\mu}=\frac{d}{d \tau}\left(b^{\mu} \kappa\right), \\
\delta \psi^{\mu} & =\frac{i}{e} \epsilon^{\mu \nu \rho \varsigma} b_{\nu} z_{\rho} \psi_{\varsigma} \kappa, \quad \delta \chi=0,
\end{aligned}
$$

with an even parameter $\kappa(\tau)$. The equations of motion have the form

$$
\begin{aligned}
& \frac{\delta S}{\delta x^{\mu}}=\frac{d}{d \tau}\left[\frac{1}{e} z_{\mu}\right]=0, \frac{\delta S}{\delta e}=\frac{1}{2 e^{2}} z^{2}=0, \frac{\delta S}{\delta b^{\mu}}=\frac{1}{e} z^{\nu}\left(\epsilon_{\nu \mu \rho \varsigma} \psi^{\rho} \psi^{\varsigma}-\frac{i \alpha}{2} g_{\mu \nu}\right)=0 \\
& \frac{\delta_{r} S}{\delta \chi}=\frac{i}{e} z_{\mu} \psi^{\mu}=0, \frac{\delta_{r} S}{\delta \psi^{\mu}}=2 i \dot{\psi}_{\mu}-\frac{1}{e} z^{\rho}\left(i g_{\mu \rho} \chi+2 \epsilon_{\mu \rho \nu \varsigma} b^{\nu} \psi^{\varsigma}\right)=0 .
\end{aligned}
$$

Going over to the Hamiltonian formulation, we introduce the canonical momenta

$$
\begin{aligned}
& \pi_{\mu}=\frac{\partial L}{\partial \dot{x}^{\mu}}=-\frac{1}{e} z_{\mu}, \quad P_{e}=\frac{\partial L}{\partial \dot{e}}=0, \\
& P_{\chi}=\frac{\partial_{r} L}{\partial \dot{\chi}}=0, \quad P_{\mu}=\frac{\partial_{r} L}{\partial \dot{\psi}^{\mu}}=-i \psi_{\mu}, \quad P_{b_{\mu}}=\frac{\partial L}{\partial \dot{b}^{\mu}}=0 .
\end{aligned}
$$

It follows from (11) that there exist primary constraints

$$
\Phi_{1}^{(1)}=P_{e}, \quad \Phi_{2}^{(1)}=P_{\chi}, \quad \Phi_{3 \mu}^{(1)}=P_{\mu}+i \psi_{\mu}, \quad \Phi_{4 \mu}^{(1)}=P_{b_{\mu}}
$$

We construct the total Hamiltonian $H^{(1)}$ according to the standard procedure (we are using the notation of Ref. [12), $H^{(1)}=H+\lambda_{A} \Phi_{A}^{(1)}$, and get, for the $H$,

$$
H=-\frac{e}{2} \pi^{2}+i \pi_{\mu} \psi^{\mu} \chi-\left(\epsilon_{\nu \mu \rho \varsigma} \pi^{\mu} \psi^{\rho} \psi^{\varsigma}+\frac{i \alpha}{2} \pi_{\nu}\right) b^{\nu} .
$$

From the conditions of the conservation of the primary constraints in time $\tau, \dot{\Phi}^{(1)}=$ $\left\{\Phi^{(1)}, H^{(1)}\right\}=0$, we find secondary constraints

$$
\Phi_{1}^{(2)}=\pi^{2}, \quad \Phi_{2}^{(2)}=\pi_{\mu} \psi^{\mu}, \quad \Phi_{3 \mu}^{(2)}=T_{\mu}=\epsilon_{\mu \nu \rho \varsigma} \pi^{\nu} \psi^{\rho} \psi^{\varsigma}+i \frac{\alpha}{2} \pi_{\mu}
$$

and determine $\lambda$, which correspond to the primary constraint $\Phi_{3}^{(1)}$. Thus, the Hamiltonian $H$ appears to be proportional to the constraints, as one could expect in the case of a reparametrization invariant theory. No more secondary constraints arise from the Dirac procedure, and the Lagrangian multipliers, corresponding to the primary constraints $\Phi_{1}^{(1)}, \Phi_{2}^{(1)}, \Phi_{4}^{(1)}$, remain undetermined. One can go over from the initial set of constraints $\left(\Phi^{(1)}, \Phi^{(2)}\right)$ to the equivalent one $\left(\Phi^{(1)}, \widetilde{\Phi}^{(2)}\right)$, where $\widetilde{\Phi}^{(2)}=\left.\Phi^{(2)}\right|_{\psi \rightarrow \widetilde{\psi}=\psi+\frac{i}{2} \Phi_{3}^{(1)}}$. The new 
set of constraints can be explicitly divided in a set of the first-class constraints, which is $\left(\Phi_{1,2}^{(1)}, \Phi_{4}^{(1)}, \widetilde{\Phi}^{(2)}\right)$ and in a set of second-class constraints, $\Phi_{3}^{(1)}$. Thus, we are dealing with a theory with first-class constraints. Our goal is to quantize this theory. First, we will try to impose as much as possible supplementary gauge conditions to perform canonical quantization. It turns out to be possible to impose supplementary gauge conditions to all the first-class constraints, excluding the constraint $\widetilde{\Phi}_{3}^{(2)}$, that corresponds to a fixation of gauge freedom which corresponds to the two type gauge transformation (7) and (8). As a result we will have only a set of first-class constraints, which is a reduction of $\Phi_{3}^{(2)}$ to the rest of constraints. These constraints we suppose to use to specify the physical states. All other constraints will be of secondclass and will be used to form Dirac brackets for canonical quantization. Thus, let us impose the gauge conditions $\Phi^{G}=0$, where

$$
\Phi_{1}^{G}=e+\zeta \pi_{0}^{-1}, \Phi_{2}^{G}=\chi, \Phi_{3 \mu}^{G}=b_{\mu}, \Phi_{4}^{G}=x_{0}-\zeta \tau, \Phi_{5}^{G}=\psi^{0}
$$

where $\zeta=-\operatorname{sgn} \pi_{0}$. (The gauge $x_{0}-\zeta \tau=0$ was first proposed in Refs. [12] as a conjugated gauge condition to the constraint $\pi^{2}=m^{2}$ in the case of scalar and spinning particles. In contrast with the gauge $x_{0}=\tau$, which together with the continuous reparametrization symmetry breaks the time reflection symmetry, and therefore fixes the variables $\zeta$, the former gauge breaks only the continuous symmetry, so that the variable $\zeta$ remains in the theory to describe states of particles $\zeta=+1$ and states of antiparticles $\zeta=-1$. Namely this circumstance allowed one to get Klein-Gordon and Dirac equations as Schrödinger ones in the course of the canonical quantization.) The requirement of consistency of the constraint $\Phi^{G}, \dot{\Phi}^{G}=0$ leads to the determination of the Lagrangian multipliers, which correspond to the primary constraints $\Phi_{1}^{(1)}, \Phi_{2}^{(1)}$ and $\Phi_{4}^{(1)}$.

To go over to a time-independent set of constraints we introduce the variable $x_{0}^{\prime}, x_{0}^{\prime}=$ $x_{0}-\zeta \tau$, instead of $x_{0}$, without changing the rest of the variables. That is a canonical transformation in the space of all variables with the generating function $W=x_{0} \pi_{0}^{\prime}+\tau\left|\pi_{0}^{\prime}\right|+W_{0}$, where $W_{0}$ is the generating function of the identity transformation with respect to all variables except $x_{0}$ and $\pi_{0}$. The transformed Hamiltonian $H^{(1)^{\prime}}$ is of the form $H^{(1)^{\prime}}=$ 
$H^{(1)}+\partial W / \partial \tau=H+\{\Phi\}$, where $\{\Phi\}$ are terms proportional to the constraints and $H$ is the physical Hamiltonian,

$$
H=\omega=|\boldsymbol{\pi}|, \quad \boldsymbol{\pi}=\left(\pi_{k}\right), \quad k=1,2,3 .
$$

One can present all the constraints of the theory (including the gauge conditions), after the canonical transformations, in the following equivalent form: $K=0, \phi=0, T=0$,

$$
\begin{gathered}
K=\left(e-\omega^{-1}, P_{e}, \chi, P_{\chi}, b^{\mu}, P_{b_{\mu}}, x_{0}^{\prime},\left|\pi_{0}\right|-\omega, \psi^{0}, P_{0}\right), \\
\phi=\left(\psi^{\|}, P_{k}+i \psi_{k}\right), T_{0}=\epsilon_{j k l} \pi_{j} \psi^{k \perp} \psi^{l \perp}-\frac{i \alpha}{2} \zeta \omega, T_{k}=-\zeta \omega \epsilon_{k j l} \psi^{j \perp} \psi^{l \perp}+\frac{i \alpha}{2} \pi_{k} .
\end{gathered}
$$

[We are using the notations $a^{k \perp}=\Pi_{i}^{k}(\boldsymbol{\pi}) a^{i}, a^{\|}=\pi_{k} a^{k}, \Pi_{i}^{k}(\boldsymbol{\pi})=\delta_{i}^{k}-\omega^{-2} \pi_{k} \pi_{i}$. Both sets of constraints $K$ and $\phi$ are of second-class, only $T$ is a first-class constraint. The set $K$ has the so called special form [12. In this case if we eliminate the variables $e, P_{e}, \chi, P_{\chi}, b^{\mu}, P_{b_{\mu}}, x^{\prime}{ }_{0},\left|\pi_{0}\right|, \psi^{0}$, and $P_{0}$ using the above constraints, the Dirac brackets for the rest of variables with respect to all second-class constraints $(K, \phi)$ reduce to ones with respect to the constraints $\phi$ only. Thus, one can only consider the variables $x^{i}, \pi_{i}, \zeta, \psi^{k}, P_{k}$, and two sets of constraints, second-class one $\phi$ and first-class one $T$. Nonzero Dirac brackets between all the variables have the form

$$
\begin{aligned}
& \left\{x^{k}, \pi_{j}\right\}_{D(\phi)}=\delta_{j}^{k}, \quad\left\{x^{k}, x^{j}\right\}_{D(\phi)}=\frac{i}{\omega^{2}}\left[\psi^{k \perp}, \psi^{j \perp}\right]_{-}, \\
& \left\{x^{k}, \psi^{j \perp}\right\}_{D(\phi)}=-\frac{\psi^{k \perp} \pi_{j}}{\omega^{2}}, \quad\left\{\psi^{k \perp}, \psi^{j \perp}\right\}_{D(\phi)}=-\frac{i}{2} \Pi_{j}^{k}(\boldsymbol{\pi}) .
\end{aligned}
$$

Thus, on this stage we have a theory with only first-class constraints $T$. These constraints are quadratic in the fermionic variables. On the one hand, that circumstance makes it difficult to impose a conjugated gauge condition, on the other hand, imposing these constraints on states vectors does not create problems with Hilbert space construction since the corresponding operators of the constraints have discrete spectra. Thus, we suppose to treat only the constraints $T$ in sense of the Dirac method. Namely, commutation relations between the operators $\hat{x}^{k}, \hat{\pi}_{k}, \hat{\psi}^{k}$, which are related to the corresponding classical variables, we calculate by means of Dirac brackets $(18)$, so that the nonzero commutators are 


$$
\begin{aligned}
& {\left[\hat{x}^{k}, \hat{\pi}_{j}\right]_{-}=i \delta_{j}^{k}, \quad\left[\hat{x}^{k}, \hat{x}^{j}\right]_{-}=-\frac{1}{\hat{\omega}^{2}}\left[\hat{\psi}^{k \perp}, \hat{\psi}^{j \perp}\right]_{-},} \\
& {\left[\hat{x}^{k}, \hat{\psi}^{j \perp}\right]_{-}=-i \frac{\hat{\psi}^{k \perp} \hat{\pi}_{j}}{\hat{\omega}^{2}},\left[\hat{\psi}^{k \perp}, \hat{\psi}^{j \perp}\right]_{+}=\frac{1}{2} \Pi_{j}^{k}(\hat{\boldsymbol{\pi}}) .}
\end{aligned}
$$

We assume also the operator $\hat{\zeta}$ to have the eigenvalues $\zeta= \pm 1$ by analogy with the classical theory, so that $\hat{\zeta}^{2}=1$. One can construct the realization of the algebra (19), above mentioned operator equation for $\hat{\zeta}$ and equations of constraints $\hat{\psi}^{\|}=0, \hat{P}_{k}+i \hat{\psi}_{k}=0$ in a Hilbert space $\mathcal{R}$, whose elements $\mathbf{f} \in \mathcal{R}$ are four-component columns depending on $\mathbf{x}$,

$$
\mathbf{f}=\left(\begin{array}{c}
f_{1}(\mathbf{x}) \\
f_{2}(\mathbf{x})
\end{array}\right),
$$

so that $f_{1}(\mathbf{x})$ and $f_{2}(\mathbf{x})$ are two- component columns. Such a realization can be found in a similar way to one used for a spinning particle with $m \neq 0$ [12]. It has the form

$$
\hat{x}^{k}=x^{k} \mathbf{I}+\frac{1}{2 \hat{\omega}^{2}} \epsilon^{k j l} \hat{\pi}_{j} \Sigma^{l}, \quad \hat{\pi}_{k}=\hat{p}^{k} \mathbf{I}, \quad \hat{\psi}^{k \perp}=\frac{1}{2} \Pi_{j}^{k}(\hat{\boldsymbol{\pi}}) \Sigma^{j}, \quad \hat{\zeta}=\gamma^{0}=\left(\begin{array}{cc}
I & 0 \\
0 & -I
\end{array}\right),
$$

where $\hat{p}^{k}=-i \partial_{k} ; I$ and $\mathbf{I}$ are $2 \times 2$ and $4 \times 4$ unit matrices; $\boldsymbol{\Sigma}=\operatorname{diag}(\boldsymbol{\sigma}, \boldsymbol{\sigma}), \sigma^{k}$ are Pauli matrices and $\gamma^{0}$ is the zeroth $\gamma$ matrix. The operators $\hat{T}$, which correspond to the first-class constraints (17), have the following form in this realization

$$
\hat{T}_{0}=\frac{i}{2}\left(\boldsymbol{\Sigma} \hat{\mathbf{p}}-\alpha \gamma^{0} \hat{\omega}\right), \hat{T}_{k}=-\hat{p}^{k} \frac{i}{2} \frac{\gamma^{0}}{\hat{\omega}}\left(\boldsymbol{\Sigma} \hat{\mathbf{p}}-\alpha \gamma^{0} \hat{\omega}\right) .
$$

Physical state vectors have to obey the conditions

$$
\hat{T}_{\mu} \mathbf{f}=0
$$

Their evolution in "time" $\tau$ is defined by the Schrödinger equation $(i \partial / \partial \tau-\hat{H}) \mathbf{f}=0$, which written in terms of the physical time $x^{0}=\zeta \tau$ (see [12]), has the form

$$
\left(i \partial_{0}-\gamma^{0} \hat{\omega}\right) \mathbf{f}(x)=0, \partial_{0}=\frac{\partial}{\partial x^{0}}, x=\left(x^{0}, \mathbf{x}\right)
$$

To find a connection of the quantum mechanics constructed with the theory of Weyl particle let us do the unitary Foldy-Wouthuysen transformation [17], adapted to the case $m=0$ : 


$$
\mathbf{f}(x)=\mathcal{U} \Psi(x), \quad \mathcal{U}=\frac{\hat{\omega}+\gamma \hat{\mathbf{p}}}{\hat{\omega} \sqrt{2}}, \quad \mathcal{U}^{\dagger} \mathcal{U}=1
$$

By straightforward calculations we get

$$
\begin{aligned}
& \check{T}=\mathcal{U}^{\dagger} \hat{T}_{0} \mathcal{U}=\frac{i}{2}\left(\gamma^{5}-\alpha\right) \gamma^{0} \gamma^{k} \partial_{k} \\
& \check{T}_{j}=\mathcal{U}^{\dagger} \hat{T}_{k} \mathcal{U}=-\frac{i}{2}\left(\gamma^{5}-\alpha\right) \partial_{k} \\
& \mathcal{U}^{\dagger}\left(i \partial_{0}-\gamma^{0} \hat{\omega}\right) \mathcal{U}=i \gamma^{0} \gamma^{\mu} \partial_{\mu}
\end{aligned}
$$

Thus, after the transformation (24) we get the Dirac equation (2) as a consequence of the Schrödinger equation (23). Conditions $\check{T}_{\mu} \Psi(x)=0$, which are consequences of (22), can be rewritten in the following form for the solutions of the Dirac equation

$$
\partial_{\mu}\left(\gamma^{5}-\alpha\right) \Psi(x)=0
$$

Let us chose $\alpha=1$. Then it follows from (26) that

$$
\Psi(x)=\left(\begin{array}{c}
u(x) \\
C
\end{array}\right),
$$

where $C$ is a constant two-component spinor and $u(x)$ a two-component spinor. To provide a finite norm of the wave function (27), we have to select $C=0$. Thus, on normalized functions $\Psi(x)$ the Eq. (26) with $\alpha=1$ is equivalent to the Weyl condition (3) for right neutrino. We have similar situation in the case $\alpha=-1$, which corresponds to the left neutrino. So, the action (6) with $\alpha=1$ describes the right neutrino and with $\alpha=-1$ describes the left neutrino.

One can also verify that formal Dirac's quantization of the action (6), without any gauge fixing, leads to the same result. In this case we have only one set of second-class constraints $\Phi_{3}^{(1)}$, which defines Dirac brackets and commutation relations. For essential operators and nonzeroth commutators we have

$$
\left[\hat{x}^{\mu}, \hat{\pi}_{\nu}\right]_{-}=i\left\{x^{\mu}, \pi_{\nu}\right\}_{D\left(\Phi_{3}^{(1)}\right)}=i \delta_{\nu}^{\mu}, \quad\left[\hat{\psi}^{\mu}, \hat{\psi}^{\nu}\right]_{+}=i\left\{\psi^{\mu}, \psi^{\nu}\right\}_{D\left(\Phi_{3}^{(1)}\right)}=-\frac{1}{2} g^{\mu \nu}
$$


As a realization of these commutation relations one can select in the form $\hat{\psi}^{\mu}=\frac{i}{2} \gamma^{\mu}, \quad \hat{x}^{\mu}=$ $x^{\mu} \mathbf{I}, \quad \hat{\pi}_{\mu}=-i \partial_{\mu}$. According to Dirac, the operators of all the first-class constraints being applied to the state vectors define the physical states. Using the primary first-class constraints $\Phi_{1,2,4}^{(1)}$ in this way, one can see that physical vectors are only functions on $x$. The operators of the secondary first-class constraints $\hat{\Phi}^{(2)}$, being applied to the state vectors, give the equations $\hat{\pi}^{2} \Psi(x)=0, \hat{\pi}_{\mu} \gamma^{\mu} \Psi(x)=0, \hat{T}_{\mu} \Psi(x)=0$. They are equivalent to two sets of independent equations $\hat{\pi}_{\mu} \gamma^{\mu} \Psi(x)=0, \hat{\pi}_{\mu}\left(\gamma^{5}-\alpha\right) \Psi(x)=0$, which are just Dirac equation (2) and the condition (26). Therefore, both ways of quantization for the action (6) give the same result.

The authors would like to thank Professor J. Frenkel for discussions and one of them (I.V.T.) is grateful to Departamento de Física Matemática da Universidade de São Paulo for the kind hospitality and to FAPESP for its support. A.E.G. was supported by CAPES. 


\section{REFERENCES}

[1] F.A. Berezin and M.S. Marinov, Pis'ma Zh. Eksp. Teor. Fiz. 21, 678 (1975) [JETP Lett. 21320 (1975)]; Ann. Phys.(NY) 104, 336 (1977).

[2] R. Casalbuoni, Nuovo Cim. A33, 115 (1976).

[3] A. Barducci, R. Casalbuoni and L. Lusanna, Nuovo Cim. A35, 377 (1976).

[4] L. Brink, S. Deser, B. Zumino, P. Di Vecchia and P. Howe, Phys. Lett. B64, 435 (1976).

[5] L. Brink, P. Di Vecchia and P. Howe, Nucl. Phys. B118, 76 (1977).

[6] A.P. Balachandran, P. Salomonson, B. Skagerstam and J. Winnberg, Phys. Rev. D15, 2308 (1977).

[7] A. Barducci and L. Lusanna, Nuovo Cim. A77, 39 (1983); J. Phys. A16, 1993 (1983).

[8] J. Gomis, M. Novell and K. Rafanelli, Phys. Rev. D34, 1072 (1986).

[9] P. Howe, S. Penati, M. Pernici and P. Townsend, Phys. Lett. B215, 555 (1988).

[10] P. Howe, S. Penati, M. Pernici and P. Townsend, Class. Quant. Grav. 6, 1125 (1989).

[11] P.A.M. Dirac, Lectures on Quantum Mechanics (Yeschiva University, 1964).

[12] D.M. Gitman and I.V. Tyutin, Class. Quantum Grav. 7, 2131 (1990); Quantization of Fields with Constraints (Springer, Berlin, 1990).

[13] G.V. Grigoryan and R.P. Grigoryan, Yad. Fiz. 53, 1737 (1991) [Sov. J. Nucl. Phys. 53, $1062(1991)]$.

[14] D.M Gitman, A.E. Gonçalves and I.V. Tyutin, Report No. IFUSP/P-1087 (1993) Int.J.Mod.Phys. (1994) to be published.

[15] A. Barducci, R. Casalbuoni, D. Dominici and L. Lusanna, Phys. Lett. B100, 126 (1981).

[16] D.M Gitman, A.E. Gonçalves and I.V. Tyutin, Report No. IFUSP/P-1094 (1993) (un- 
published).

[17] L.L. Foldy and S.A. Wouthuysen, Phys. Rev. 78, 29 (1950). 